\title{
Development of intron polymorphism markers in major latex-like protein gene for locality-level and cultivar identification of Salvia miltiorrhiza
}

\author{
Hongtao Wang ${ }^{1}$, Na Hao ${ }^{1}$, Lei Chen ${ }^{1}$ and Guisheng Li ${ }^{2}$
}

\begin{abstract}
Background: Salvia miltiorrhiza (Danshen) is one of the most widely used medicinal herbs in traditional Chinese medicine. Locality-level and cultivar identification is of great importance not only for protecting highest therapeutic effectiveness of Daodi Danshen, but also for the genetic conservation and utilization of existing S. miltiorrhiza populations.

Results: Intron polymorphisms including SNPs (single nucleotide polymorphisms) and indels were exploited in major latex-like protein (MLP) gene. Based on these markers, genetic relationships among S. miltiorrhiza cultivar and populations in different locations were evaluated by constructing a dendrogram. Moreover, S. miltiorrhiza specimens from Laiwu region were geographically distinguishable by the developed SNP marker. A 204 bp-indel marker was exploited for the first space breeding cultivar Luyuan Danshen-1 (LD-1), and an effective real-time PCR assay was successfully developed for fast screening of LD-1 among local landraces.
\end{abstract}

Conclusions: MLP intron is a valuable DNA barcode for intra-specific study of S. miltiorrhiza populations, and the developed markers can serve as a useful tool for molecular identification of LD-1 cultivar and geographically distinct populations of S. miltiorrhiza.

Keywords: Salvia miltiorrhiza, Locality-level identification, Cultivar identification, Major latex-like protein, Intron polymorphism

\section{Background}

Salvia miltiorrhiza (Lamiaceae), known as Danshen in Chinese, is one of the most widely used medicinal herbs in traditional Chinese medicine. Its dried root or rhizome, commonly called 'red sage', has been used for centuries in the treatment of cardiovascular and cerebrovascular diseases (Zhou et al. 2005). S. miltiorrhiza contains two major groups of active compounds: the lipophilic diterpenoids and hydrophilic phenolic acids ( $\mathrm{Li}$ et al. 2009). Modern pharmacological studies have demonstrated that these compounds are responsible for many

\footnotetext{
*Correspondence: Isyq_003@163.com

${ }^{2}$ School of Pharmacy, College of Pharmacy, Yantai University, Yantai, People's Republic of China

Full list of author information is available at the end of the article
}

therapeutic actions, such as anti-inflammatory, antibacterial, anti-carcinogenic, and antioxidant activities (Zhou et al. 2005; Hung et al. 2010; Wang 2010). Besides, $S$. miltiorrhiza has also become widely accepted as functional food due to its significant antioxidant capacity.

The Chinese term 'Daodi' refers to the medicinal material that is produced in specific geographic regions with designated natural conditions and ecological environment. The superior germplasm resource and suitable geographic regions are the key factors of the formation of Daodi medicinal material (Zhao et al. 2012). Shandong province is one of the most important Daodi production regions in China and has rich germplasm resources of S. miltiorrhiza (Song et al. 2010). Due to the decrease of wild resources, S. miltiorrhiza landraces of different 
production areas in Shandong province have become the major source of commercial Danshen herb. Nowadays, with the rapid development of space technologies, space breeding provides a new technical platform for screening new varieties of crops. Compared to the plant growing conditions on Earth, the effects of space environment, such as high-energy ion radiation, microgravity, space magnetic field, ultra vacuum, offer great opportunities of genetic mutagenesis. In 2004, the dried seeds of S. miltiorrhiza were carried into space by China's 20th retrievable satellite. After 18 days' spaceflight with retrievable satellite, the returned seeds were screened by ground-based observation and selection. Luyuan Danshen-1 (LD-1) is the first successfully selected space breeding cultivar of S. miltiorrhiza, which was registered in Shandong Provincial Department of Agriculture in 2013. LD-1 cultivar produces a higher content of salvianolic acid and yield than local landraces. Besides, S. miltiorrhiza from different geographic populations have been shown to contain different contents of active compounds (Ran et al. 2008). For example, S. miltiorrhiza from Laiwu region was regarded to be more potent than those grown in other regions. Therefore, locality and cultivar identification is very important not only for protecting the quality of Shandong Daodi Danshen, but also for genetic conservation and utilization of existing S. miltiorrhiza populations.

Traditional means for authentication of S. miltiorrhiza populations commonly rely on morphological characteristics. These approaches, however, are sometimes unreliable because most of $S$. miltiorrhiza populations and cultivar are morphologically similar (Zhang et al. 2013), which renders their differentiation subjective and errorprone. In recent years, various molecular markers have been developed for investigating the genetic diversity of S. miltiorrhiza, including RAPD (Guo et al. 2002), AFLP (Wang et al. 2007), CoRAP (Wang et al. 2009), ISSR (Zhang et al. 2013), and SRAP (Song et al. 2010). While previous studies focused on the population structure and rich genetic diversity within cultivated populations in different producing areas of China, no research attention has been paid to the cultivar and locality-level identification of S. miltiorrhiza. In this study, we aimed to develop intron polymorphism markers in major latex-like protein $(M L P)$ gene to evaluate genetic relationships among $S$. miltiorrhiza populations in different locations, and constructed a simple method for cultivar and locality-level discrimination of S. miltiorrhiza in Shandong province.

\section{Results}

PCR of $M L P$ intron and sequence analysis

In order to check the feasibility of the designed primers $\mathrm{mlpF}$ and mlpR, seven S. miltiorrhiza samples from four different regions were randomly chosen for amplification of $M L P$ intron. Figure 1 showed that all the samples were successfully amplified with their single bands, except that the LD-1 cultivar generated two fragments. $M L P$ introns of the $120 \mathrm{~S}$. miltiorrhiza samples were amplified and sequenced, and a total of 10 genotypes were detected. As shown in Additional file 1: Fig. S1, two genotypes were discovered respectively in $S$. miltiorrhiza (smL1, smL2) and S. miltiorrhiza f. alba (smf1, smf2) in Laiwu, two genotypes were detected in Weifang population (smW1, smW2) and LD-1 cultivar (H41, H42), and samples in Yantai (smYt) and Linyi (smLy) showed their unique genotypes. The compiled DNA sequences were registered in GenBank with accession numbers of KU891682KU891691. Multiple sequence alignment results showed that intron length polymorphisms and SNPs were exploited in $M L P$ intron. As shown in Fig. 2 and Additional file 1: Fig. S1, a $204 \mathrm{bp}$-insertion was determined in the longer fragments (H41) of LD-1, compared with the shorter band (H42) and fragments of other landrace samples. Besides, at the 562 bp nucleotide position of Additional file 1: Fig. S1, a SNP unique to Laiwu population was discovered. Samples from Laiwu region contain nucleotide $\mathrm{T}$, which was replaced by $\mathrm{C}$ at the same position in the samples from other geographic regions.

\section{Genetic relationship among different populations and specific primer design}

The polymorphic $M L P$ intron sequences including SNPs and indels of different $S$. miltiorrhiza populations and cultivar were used to construct a neighbor-joining dendrogram. As shown in Fig. 3, the LD-1 cultivar (H41 type) formed a separate clade showing less similarity with the other populations, indicating that the space-induced mutagenesis was indeed occurred in LD-1. The sister large group was divided into two subclusters: one consisted of samples of Weifang (SMW2 type), Laiwu (SML2

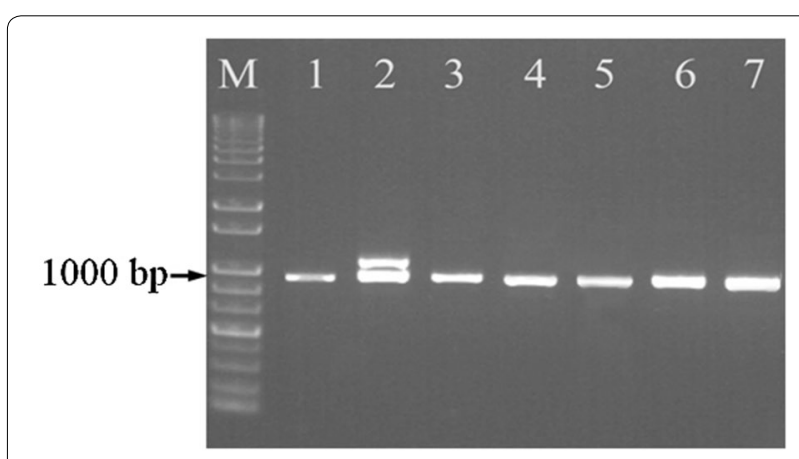

Fig. 1 PCR products of MLP introns of S. miltiorrhiza samples from different regions. Lane M 1000 bp DNA ladder, lane 1 Yantai, lane 2 LD-1, lane 3 Linyi, lanes 4, 5, Laiwu, lanes 6, 7 Weifang 


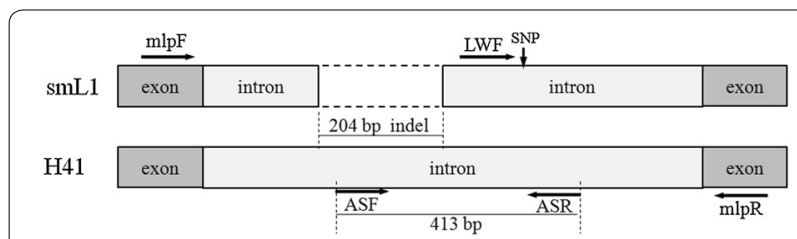

Fig. 2 Graphical overview of the positions of the primer sets used in this study

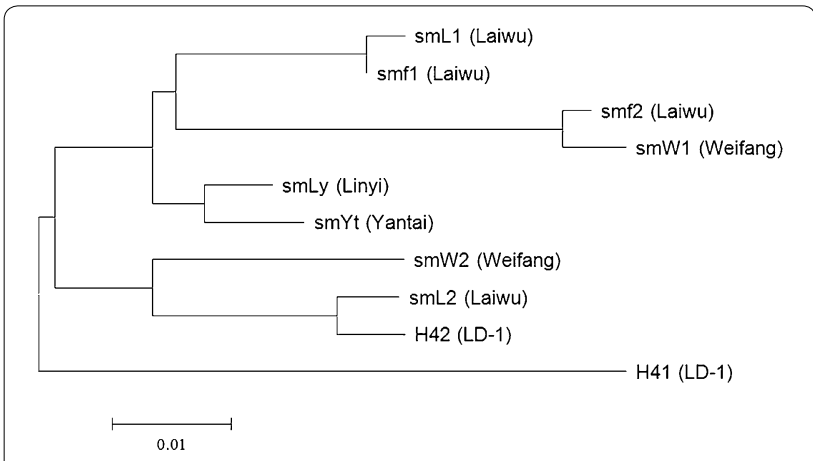

Fig. 3 Dendrogram constructed on the basis of MLP intron sequences using the neighbor-joining method

type), and LD-1 cultivar (H42 type), and the other branch contained specimens from Laiwu (SML1 type), Weifang (SMW2 type), Linyi, and Yantai. These results demonstrated that the landrace from which LD-1 cultivar originated has a close relationship with the samples in Laiwu (smL2), and there were gene exchange between populations of Laiwu and Weifang.

To authenticate $S$. miltiorrhiza populations originated from Laiwu and the LD-1 cultivar, specific primers were designed based on their unique sequences. Primer LWF (5'-CATACCCACTATACATTCTTGAT-3') was designed for specific identification of $S$. miltiorrhiza from Laiwu, by substituting of $\mathrm{G}$ for $\mathrm{A}$ at the third base from $3^{\prime}$ end. Primers ASF (5'-CTTTACTTTCGGC ACTGGTT- ${ }^{\prime}$ ) and ASR (5'-ATCCGTCTCACTTA TCTTGG-3') were designed for specific authentication of LD-1from other local landraces. The relative positions of these primers were shown in Fig. 2 and Additional file 1: Fig. S1.

\section{Molecular authentication of Laiwu population and LD-1}

Molecular authentication of S. miltiorrhiza from Laiwu population was performed using multiplex PCR. As shown in Fig. 4, all the samples generated the same fragments as shown in Fig. 1, but only those from Laiwu produced their specific bands representing the $\mathrm{T}$ allele. Similarly, primers ASF and ASR amplified LD-1-specific band of $413 \mathrm{bp}$, but no PCR products were detected in

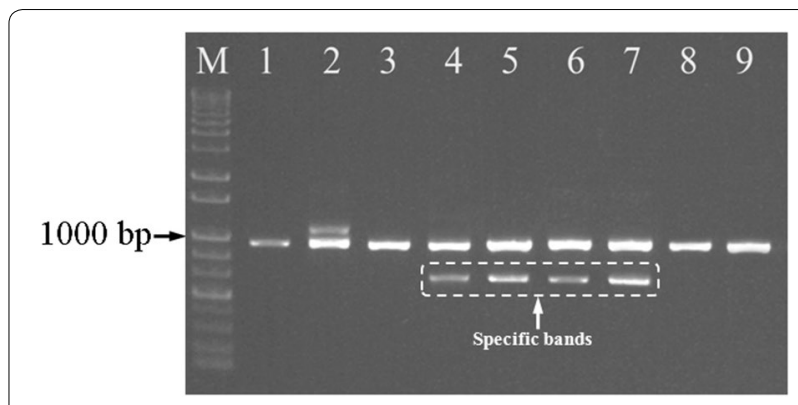

Fig. 4 Multiplex PCR products using primers mlpF, mlpR, and LWF. Lane M 1000 bp DNA ladder, lane 1 Yantai, lane 2 LD-1, lane 3 Linyi, lanes 4-7 Laiwu, lanes 8, 9 Weifang

other landraces (Fig. 5). In order to check the accuracy of the indel marker and construct a fast assay of LD-1, real time PCR was conducted and endpoint analysis method was used for identification of LD-1. Figure 6 showed that 40 LD-1 samples were easily discriminated from other landraces by their signal levels compared with the positive threshold. Therefore, LD-1 cultivar and S. miltiorrhiza from Laiwu population can be successfully authenticated by the indel and SNP markers in $M L P$ intron.

\section{Discussion}

Locality-level and cultivar identification is of great importance to ensure highest therapeutic effectiveness and help select the best strains of S. miltiorrhiza. There have been a number of studies developing molecular markers for S. miltiorrhiza grown in different geographical regions (Wang et al. 2007; Song et al. 2010; Zhang et al. 2013). Although high genetic diversity was revealed among S. miltiorrhiza populations, effective localitylevel identification is difficult because molecular markers such as RAPD, ISSR, SSR, CoRAP, and SRAP require more primer pairs to obtain enough PCR products and are easily affected by PCR conditions. On the other hand,

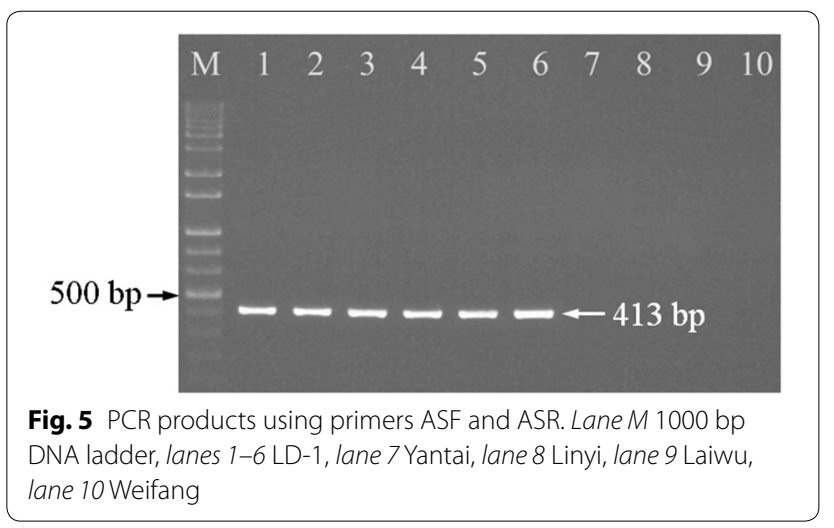




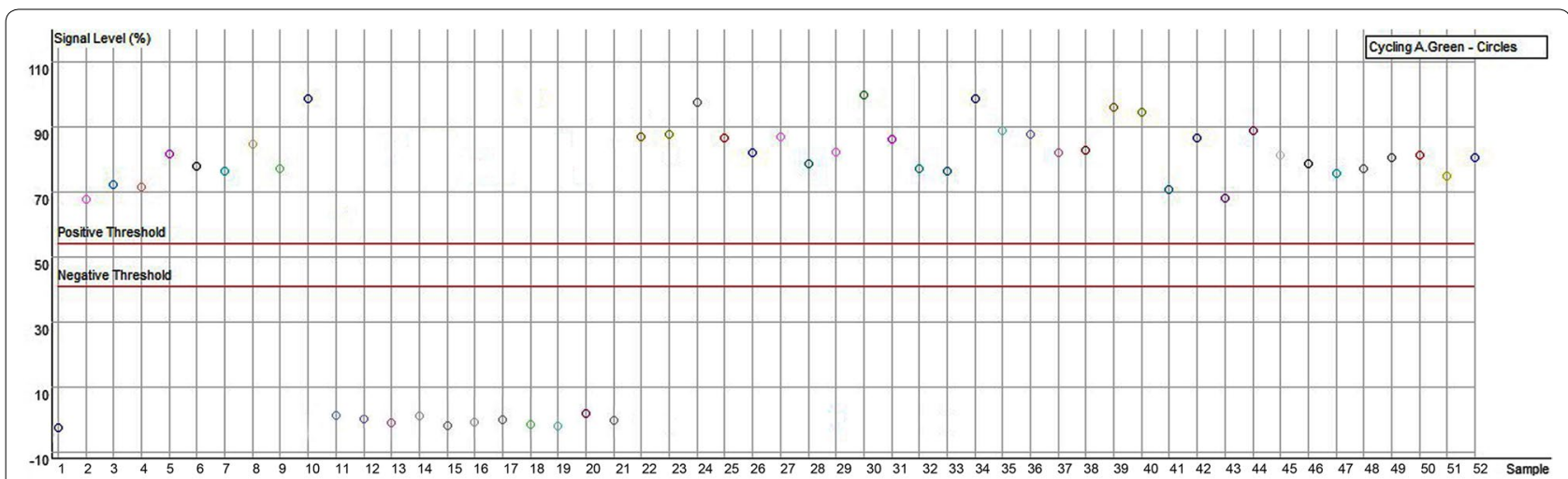

Fig. 6 Endpoint analysis for discriminating of LD-1 from other samples. 1 No template control, 21 negative control, 52 positive control, 11-14 Laiwu, 15-16 Weifang, 17-18 Linyi, 19-20 Yantai, 2-10 22-51: LD-1

commonly used DNA barcodes like ITS2 and psbA-trnH are not polymorphically informative among $S$. miltiorrhiza populations to develop SNP markers (Chen et al. 2010). In this study, intron polymorphism (SNP and indel) markers were exploited in $M L P$ gene and proved to be effective for molecular identification of LD-1 cultivar and laiwu population of S. miltiorrhiza.

A host of SNPs and indels existed in $M L P$ introns among and within different populations. The high genetic polymorphism among populations of different regions may greatly attribute to the influence of geographic environments. The high level of genetic polymorphism within populations (such as Laiwu and Weifang) may result from the traditional practice of mixed cultivation of seeds without deliberate selection. Especially in Laiwu region, S. miltiorrhiza var. miltiorrhiza and S. miltiorrhiza f. alba are often planted together in one field, where cross-pollination occurs at a high rate. It is worthy to note that a 204 bp-insertion was detected in the intron of MLP gene in cultivar LD-1, which indicated that space mutagenesis breeding is an efficient way for creating new germplasms of $S$. miltiorrhiza. The association between the indel marker and the agronomic characters of LD-1 needs further study.

Molecular authentication of S. miltiorrhiza from Laiwu was achieved by exploiting a SNP site unique to Laiwu population. S. miltiorrhiza samples from Laiwu can be easily discriminated from other populations by the specific amplicon representing $\mathrm{T}$ allele in $M L P$ intron. The established multiplex PCR was conducted many times with 20 specimens and showed $100 \%$ accuracy. Intron length is relatively stable because indels of nucleotides do not tend to occur as often as SNPs (Ching et al. 2002). An indel marker was developed for the space breeding cultivar LD-1, and the real time PCR with endpoint analysis enabled clear identification and fast screening of LD-1 from landraces of different populations. Therefore, a simple and convenient method for identification of S. miltiorrhiza populations and cultivar was developed based on the intron polymorphism markers in $M L P$ gene.

\section{Conclusions}

In this study, we demonstrated that the intron of $M L P$ gene is a valuable DNA barcode for intra-specific polymorphism exploitation of S. miltiorrhiza. The markers developed in this study can serve as a useful tool for marker-assisted selection of LD-1 cultivar and geographically distinct populations of $S$. miltiorrhiza, and the method may also be applied to closely related populations and cultivars of other medicinal plants.

\section{Methods}

\section{Plant materials and DNA isolation}

A total of 120 samples of S. miltiorrhiza were collected from four geographically distinct populations in Shandong province. All the specimens were morphologically identified by Prof. Guisheng $\mathrm{Li}$ and deposited in Star Aviation Breeding Company (Yantai, Shandong). According to their localities and variety, the plant samples were divided into six groups for analysis (Table 1). Genomic DNA of the 120 individuals were respectively isolated by using a Plant DNA isolation kit (Easypure Plant Genomic DNA Kit, TransGen Biotech), according to the manufacturer's instructions. The prepared 120 DNA samples were stored at $-20^{\circ} \mathrm{C}$ for further analysis.

\section{PCR of MLP intron and sequence analysis}

PCR amplification of $M L P$ intron were conducted using primers mlpF (5'-TTTAGGCACAAACCACATG ATC- $3^{\prime}$ ) and mlpR (5'-CGTGGGCTGTAATAACGA ATG-3'), which were designed according to the exon 
Table 1 Plant samples used in this study

\begin{tabular}{|c|c|c|c|}
\hline Variety/cultivar & Location & No. of samples & Voucher specimen \\
\hline $\begin{array}{l}\text { S. miltiorrhiza var. } \\
\text { miltiorrhiza }\end{array}$ & Yantai & 15 & SMyt01 \\
\hline S. miltiorrhiza f. alba & Laiwu & 15 & SMflw11, SMflw17 \\
\hline $\begin{array}{l}\text { S. miltiorrhiza var. } \\
\text { miltiorrhiza }\end{array}$ & Laiwu & 15 & SMlw23, SMlw26 \\
\hline $\begin{array}{l}\text { S. miltiorrhiza var. } \\
\text { miltiorrhiza }\end{array}$ & Linyi & 15 & SMly31 \\
\hline $\begin{array}{l}\text { S. miltiorrhiza var. } \\
\text { miltiorrhiza }\end{array}$ & Weifang & 20 & SMwf42, SMwf47 \\
\hline Luyuan Danshen-1 & Yantai & 40 & $\mathrm{H} 401$ \\
\hline
\end{tabular}

sequences flanking the target intron (GenBank accession GQ923782). The $20 \mu \mathrm{L}$ reaction mixture consist of $10-50 \mathrm{ng}$ of template DNA, $0.5 \mu \mathrm{M}$ of each primer, and $10 \mu \mathrm{L}$ of $2 \times$ EasyTaq PCR SuperMix (TransGen Biotech). The mixtures were heated at $94{ }^{\circ} \mathrm{C}$ for $4 \mathrm{~min}$ followed by 33 cycles of $30 \mathrm{~s}$ at $94{ }^{\circ} \mathrm{C}, 30 \mathrm{~s}$ at $60^{\circ} \mathrm{C}$, and a 1 -min extension at $72{ }^{\circ} \mathrm{C}$ with final extension reaction at $72{ }^{\circ} \mathrm{C}$ for $7 \mathrm{~min}$. PCR products were analyzed via $1.0 \%$ agarose gel electrophoresis and visualized by ethidium bromide staining under UV.

The PCR products were cut and recycled with an EasyPure Quick Gel Extraction kit (TransGen Biotech), according to the manufacturer's instructions. The purified products were ligated into the pGEM-T Easy vector (Promega, USA) and transformed into competent E. coli $\mathrm{DH} 5 \alpha$ cells. After transformant selection, white clones were cultivated in LB liquid medium at $37{ }^{\circ} \mathrm{C}$ overnight with shaking. Plasmid DNA was isolated with a Plasmid DNA MiniPrep kit (TransGen Biotech) and sequenced for both forward and reverse directions on an automatic DNA sequencer (ABI PRISM 3700, USA). DNA sequences were assembled using SeqMan software, and multiple sequence alignments were conducted using the Clustal Omega program (Sievers and Higgins 2014).

\section{Phylogenetic analysis and specific primer design}

Phylogenetic analysis was performed to construct a dendrogram using the neighbor-joining module of the MEGA software (Tamura et al. 2011). Based on the SNP site representing Laiwu population, primer was designed by introducing an additional mismatch for specific identification of S. miltiorrhiza from Laiwu population (Drenkard et al. 2000). Primers specific to LD-1 were designed according to the insertion sequence compared to the other populations.
Molecular authentication of Laiwu population and LD-1 Molecular authentication of Laiwu population was conducted with primers mlpF, mlpR, and LWF. Primers mlpF and mlpR were included in all reactions and served to provide a control PCR product. PCR was performed in a total volume of $20 \mu \mathrm{L}$, and the reaction mix consisted of each of the primers at a concentration of $0.5 \mu \mathrm{M}, 10-50 \mathrm{ng}$ of template DNA, and $10 \mu \mathrm{L}$ of $2 \times$ EasyTaq PCR SuperMix (TransGen Biotech). The PCR amplification profile was identical with described above. Molecular identification of LD-1 cultivar was performed with primers ACF and ACR. The $20 \mu \mathrm{L}$ reaction mixture consist of $10-50 \mathrm{ng}$ of template DNA, $0.5 \mu \mathrm{M}$ of each primer, and $10 \mu \mathrm{L}$ of $2 \times$ EasyTaq PCR SuperMix (TransGen Biotech). The mixtures were heated at $94{ }^{\circ} \mathrm{C}$ for $4 \mathrm{~min}$ followed by 33 cycles of $30 \mathrm{~s}$ at $94{ }^{\circ} \mathrm{C}, 30 \mathrm{~s}$ at $62{ }^{\circ} \mathrm{C}$, and a 1 -min extension at $72{ }^{\circ} \mathrm{C}$ with final extension reaction at $72{ }^{\circ} \mathrm{C}$ for $7 \mathrm{~min}$. To validate the accuracy of the indel marker and construct a fast assay of LD-1, a real-time PCR was conducted. The $10 \mu \mathrm{L}$ reaction mixture consisted of 5-50 ng DNA, $5 \mu \mathrm{M}$ of each primer, and $5 \mu \mathrm{L} 2 \times$ SYBR Green I Mastermix (SensiMixPlus SYBR, Australia). The PCR cycling profile was as follows: 10 min of activation at $95^{\circ} \mathrm{C}$, followed by 40 cycles of a three-step thermal profile involving $10 \mathrm{~s}$ at $95{ }^{\circ} \mathrm{C}$ for denaturation, $15 \mathrm{~s}$ at $62{ }^{\circ} \mathrm{C}$ for combined annealing, and $20 \mathrm{~s}$ at $72{ }^{\circ} \mathrm{C}$ for extension. The melting analysis condition was performed with a ramp from 85 to $98^{\circ} \mathrm{C}$, rising by $1{ }^{\circ} \mathrm{C}$ at each step. Endpoint analysis method was used for the determination of LD-1.

\section{Additional file}

Additional file 1: Fig. S1. Comparison of MLP intron sequences of different populations and LD-1 cultivar.

\section{Abbreviations}

SNP: single nucleotide polymorphism; MLP: major latex-like protein; LD-1: Luyuan Danshen-1; RAPD: random amplified polymorphic DNA; AFLP: amplified fragments length polymorphism; CoRAP: conserved region amplification polymorphism; ISSR: inter-simple sequence repeat; SRAP: sequence related amplified polymorphism; SSR: simple sequence repeat; ITS: internal transcribed spacer.

\section{Authors' contributions}

HW and GL designed the experiments; HW and LC performed the experiment and analyzed the data; GL contributed reagents and materials; $\mathrm{HW}$ and $\mathrm{NH}$ wrote the paper. All authors read and approved the final manuscript.

\footnotetext{
Author details

${ }^{1}$ School of Life Sciences, Yantai University, Yantai, People's Republic of China. ${ }^{2}$ School of Pharmacy, College of Pharmacy, Yantai University, Yantai, People's Republic of China.
}

\section{Competing interests}

The authors declare that they have no competing interests. 


\section{Availability of data and materials}

The datasets supporting the conclusions of this article are available in the GenBank (National Center for Biotechnology Information) repository.

\section{Funding}

This work was financially supported by the Shandong Provincial Natural Science Foundation (ZR2013CQ021) and the Scientific Research Foundation for the Returned Overseas Chinese Scholars, State Education Ministry. The funder had no role in study design, data collection and analysis, decision to publish, or preparation of the manuscript.

Received: 30 March 2016 Accepted: 27 October 2016

Published online: 04 November 2016

\section{References}

B-I Guo, Lin S, Y-x Feng, Zhao Y-J (2002) Primary research on genetic relationship among main populations of Salvia miltiorrhiza and genuineness of herb. Chin Tradit Herb Drugs 33:1113-1116

Chen S, Yao H, Han J, Liu C, Song J, Shi L, Zhu Y, Ma X, Gao T, Pang X, Luo K, Li Y, Li X, Jia X, Lin Y, Leon C (2010) Validation of the ITS2 region as a novel DNA barcode for identifying medicinal plant species. PLoS ONE 5:e8613

Ching A, Caldwell KS, Jung M, Dolan M, Smith OS, Tingey S, Morgante M, Rafalski AJ (2002) SNP frequency, haplotype structure and linkage disequilibrium in elite maize inbred lines. BMC Genet 3:19

Drenkard E, Richter BG, Rozen S, Stutius LM, Angell NA, Mindrinos M, Cho RJ, Oefner PJ, Davis RW, Ausubel FM (2000) A simple procedure for the analysis of single nucleotide polymorphisms facilitates map-based cloning in Arabidopsis. Plant Physiol 124:1483-1492

Hung YC, Wang PW, Pan TL (2010) Functional proteomics reveal the effect of Salvia miltiorrhiza aqueous extract against vascular atherosclerotic lesions. Biochim Biophys Acta 1804:1310-1321
Li Y-G, Song L, Liu M, Hu ZB, Wang Z-T (2009) Advancement in analysis of Salviae miltiorrhizae Radix et Rhizoma (Danshen). J Chromatogr A 1216:1941-1953

Ran R, Zhou F, Wang T, Kong Q (2008) Comparison of tanshinone IIA in Radix Salviae Miltorrhizae from Shandong. J Chin Med Mater 31:331-333

Sievers F, Higgins DG (2014) Clustal Omega, accurate alignment of very large numbers of sequences. Methods Mol Biol 1079:105-116

Song Z, Li X, Wang H, Wang J (2010) Genetic diversity and population structure of Salvia miltiorrhiza Bge in China revealed by ISSR and SRAP. Genetica 138:241-249

Tamura K, Peterson D, Peterson N, Stecher G, Nei M, Kumar S (2011) MEGA5: molecular evolutionary genetics analysis using maximum likelihood, evolutionary distance, and maximum parsimony methods. Mol Biol Evol 28:2731-2739

Wang B-Q (2010) Salvia miltiorrhiza: chemical and pharmacological review of a medicinal plant. J Med Plants Res 4:2813-2820

Wang B, Zhang Y, Chen CB, Li XL, Chen RY, Chen L (2007) Analysis on genetic diversity of different Salvia miltiorrhiza geographical populations in China. Chin J Chin Mater Med 32:1988-1991

Wang Q, Zhang B, Lu Q (2009) Conserved region amplification polymorphism (CoRAP), a novel marker technique for plant genotyping in Salvia miltiorrhiza. Plant Mol Biol Rep 27:139-143

Zhang Y, Li X, Wang Z (2013) Diversity evaluation of Salvia miltiorrhiza using ISSR markers. Biochem Genet 51:707-721

Zhao Z, Guo P, Brand E (2012) The formation of daodi medicinal materials. J Ethnopharmacol 140:476-481

Zhou L, Zuo Z, Chow MS (2005) Danshen: an overview of its chemistry, pharmacology, pharmacokinetics, and clinical use. J Clin Pharmacol 45:1345-1359

\section{Submit your manuscript to a SpringerOpen ${ }^{\circ}$ journal and benefit from:}

- Convenient online submission

- Rigorous peer review

- Immediate publication on acceptance

- Open access: articles freely available online

- High visibility within the field

- Retaining the copyright to your article 